\title{
What's Mind? No matter!
}

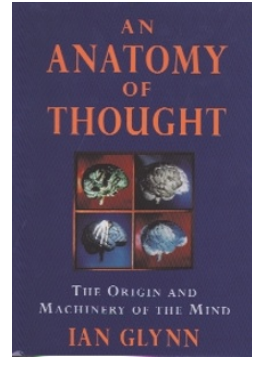

\section{An Anatomy of Thought}

by Ian Glynn

Weidenfeld \& Nicholson, London, 1999. £25

Hardcover, pp 456

ISBN 0-29782-002-8

\section{Reviewed by Michael Morgan}

Ian Glynn has written an elegant, stimulating and frequently charming book on the relationship among brain, thought and consciousness. An unusual feature of the book is that it is written by an eminent research scientist, who nonetheless respects the work of professional philosophers and has clearly read their works with close attention. This results in a final chapter on the philosophy of mind that will be a considerable intellectual challenge to non-philosophers, who will have to distinguish type/type and token/token identity theories, and contemplate Davidson's idea that "though each instance of a mental state is identical with some physical brain state, there are in general no laws describing the connections between the mental and physical characteristics."

The earlier chapters are easier going. An admirably clear introductory chapter on Darwin's theory of natural selection and its application to hominid evolution is followed by a clear account of how nervous systems encode sensory signals and exchange messages. The major kinds of sensory transduction are well covered. The chapter entitled "Cook's Tour of the Brain" teaches the major features of human brain anatomy, using evidence from electrical recording, stimulation, brain lesions and functional brain imaging (although not much of the latter). This is followed by specialized sections on vision, language and cognition (including emotions) and, finally, by the philosophical section mentioned above.

A current advertisement for a London cinema chain says "If you see only one film this year, you don't get out enough." If the general reader read only this book on neuroscience, would they have read enough? At the end of the book, one is amazed that hardly any area of modern neuroscience is left untouched. Unusually for a physiologist, Glynn even has a kind word to say about Freud, recognizing (as it is now fashionable

Michael Morgan is at the Institute of

Opthalmology, University College London, Bath Street, London EC1V 9EL, UK.

Email:m.j.morgan@ucl.ac.uk in certain quarters to deny) that Freud put unconscious motivation on the map. This would be an excellent book to attract a bright student into neuroscience. However, I have a feeling that it is just a bit demanding to compete with "Captain Corelli's Mandolin" on the beach. Equations, of course, are rigorously avoided (no mean feat when talking Hodgkin and Huxley!), but there are all kinds of other unavoidable technicalities, in chemistry and optics, for example, that will cause difficulty. However, one can never tell about these things, and I would love to see this book on the scientific best-seller list, where it deserves to be.

One rather odd feature of a book about the physical basis of sensation and consciousness is that little attention is paid to the question of how these things can be scientifically measured. The unwary reader will get the unfortunate impression that 'psychophysics' is about looking at illusions, that it started out quite promisingly in Gestalt psychology and then came to a bad end. This neglect of psychophysics undermines, in my view, the central aim in the book, as the reader is not being asked to formulate, or even to face up to, the really difficult scientific questions about consciousness. For example, the book describes an experimental result in which observers are said to have been able to base decisions upon, despite remaining unconscious of, monocular information about a target that was masked from the other eye ${ }^{1}$. Yet how do we know they were unconscious of the information? Subjects were asked both to guess the position of the target and to state their confidence on a scale of $0-9$. The crucial result was that ratings of confidence did not correlate with correct decisions; this was an excellent idea, but unfortunately observers were encouraged to use the whole scale, whatever the strength of their sensations. Because the stimulus was usually barely visible, observers were in effect being encouraged to say that they were confident when they were not. The lack of correlation of confidence with success may therefore have been a measurement $\operatorname{artifact}^{2}$. In the study of consciousness, as in all science, the devil is in the details.

I would also have liked to see more detail on the physical and statistical nature of images, hard though it may be to get across without some mathematics. The fact that we have three classes of cone receptor, not two or five, is comprehensible from an evolutionary point of view once we realize that natural spectra are only a small subset of possible spectra, and that over $95 \%$ of the variance among them can be accounted for by just three spectral basis functions ${ }^{3}$. Similarly, there are strong arguments that the Gabor-type receptive fields of cortical simple cells are a good basis set for describing natural images, which have a particular statistical structure 4 . Impressive progress is being made in using this basis set to recognize, for example, the gender of faces. The reader will not guess this from the rather antiquated presentation of such cells as 'feature detectors.' I would make the strong statement that it is not possible to understand image processing of any sort (including the biological) without the notion that images contain constrained statistical structures at different spatial scales. Yet this topic is hardly touched upon, and key names are not in the index.

What about the philosophy? Clearly we are all identity theorists now; dualists in neuroscience are about as common as Lamarkians in biology. Brain events and sensations are somehow the same. Yet are they the same in the sense that "Tony Blair" and "The Prime Minister of Britain" are the same, or in some different sense? Can all brain events be sensations? Only certain action potentials? Only certain kinds of neural circuits? Only neural circuits carrying out particular kinds of computation? Because the answer to these questions is still completely unknown, it is tempting to take a different line and ask instead why we find the whole problem so baffling. One statement of the problem that avoids a duality between mind and matter is the following. Our brains represent the world. Among these representations is something called 'the brain'. How does this representation represent other things, let alone itself? At this point, the 40amp fuse blows. A T-shirt I saw at a conference made the point admirably: "I used to think the brain was the most intricate and important organ in the body. And then I thought, hey, who's telling me this?"

\footnotetext{
1. Kolb, F. C. \& Braun, J. Nature 377, 336-338 (1995).

2. Morgan, M. J., Solomon, J. A. \& Mason, A. J. S. Nature 385, 401-402 (1997).

3. Lennie, P. \& D'Zmura, M. CRC Crit. Rev. Neurobiol. 3, 333-400 (1988).

4. Olshausen, B. A. \& Field, D. J. Nature 381, 607-609 (1996).
} 